\title{
Molecular Design Strategies for Color Tuning of Blue TADF Emitters
}

\author{
Patrycja Stachelek, ${ }^{*}{ }^{\dagger}$ Jonathan S. Ward, ${ }^{\ddagger}$ J๑ Paloma L. dos Santos, ${ }^{\dagger}$ Andrew Danos, ${ }^{\dagger}{ }^{\circledR}$ Marco Colella, ${ }^{\dagger}$
}

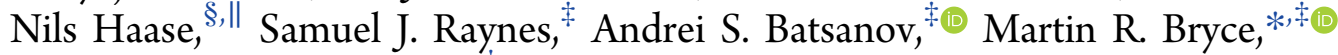
and Andrew P. Monkman* ${ }^{* \dagger}$

${ }^{\dagger}$ Department of Physics and ${ }^{\star}$ Department of Chemistry, Durham University, Durham DH1 3LE, U.K.

${ }^{\S}$ Institute of Physics, Experimental Physics IV, University of Augsburg, Universitätsstr. 1, Augsburg 86135, Germany

"Merck KGaA, Performance Materials-Display Solutions, Frankfurter Straße 250, Darmstadt 64293, Germany

Supporting Information

ABSTRACT: New thermally activated delayed fluorescence (TADF) blue emitter molecules based on the known donor-acceptor-donor (D-A-D)type TADF molecule, 2,7-bis(9,9-dimethylacridin-10-yl)-9,9-dimethylthioxanthene-S,S-dioxide (DDMA-TXO2), are reported. The motivation for the present investigation is via the use of rational molecular design, based on DDMA-TXO2, to elevate the organic light emitting diode (OLED) performance and obtain deeper blue color coordinates. To achieve this goal, the strength of the donor (D) unit and acceptor (A) units have been tuned with methyl substituents. The methyl functionality on the acceptor was also expected to modulate the $\mathrm{D}-\mathrm{A}$ torsion angle in order to obtain a blue shift in the electroluminescence. The effect of regioisomeric structures has also been investigated. Herein, we report the photophysical, electrochemical, and single-crystal X-ray crystallography data to assist with the successful

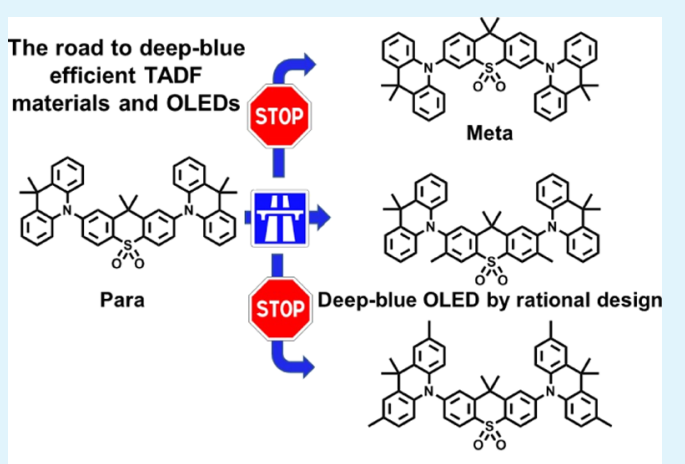

Increased donor strength OLED design. The methyl substituents on the DDMA-TXO2 framework

have profound effects on the photophysics and color coordinates of the emitters. The weak electron-donating methyl groups alter the redox properties of the $\mathrm{D}$ and $\mathrm{A}$ units and consequently affect the singlet and triplet levels but not the energy gap $\left(\Delta E_{\mathrm{ST}}\right)$. By systematically manipulating all of the aforementioned factors, devices have been obtained with acceptor-substituted III with a maximum external quantum efficiency of $22.6 \%$ and Commission Internationale de l'Éclairage coordinates of $(0.15$, $0.18)$ at $1000 \mathrm{~cd} \mathrm{~m}^{-2}$.

KEYWORDS: TADF, photophysics, blue OLEDs, charge transfer, donor/acceptor tuning

\section{INTRODUCTION}

The research on materials that exhibit thermally activated delayed fluorescence (TADF) has increased dramatically since the introduction of TADF materials into organic light-emitting diodes (OLEDs) for lighting and display applications. ${ }^{1-4}$ Although highly efficient green TADF OLED materials have been widely reported, ${ }^{5-7}$ research to create efficient and stable red and especially blue TADF OLEDs has not progressed as rapidly. ${ }^{8,9}$

By virtue of the key reverse intersystem crossing (rISC) step being thermally activated, it is now understood that the tripletharvesting efficiency of TADF materials depends critically, but not exclusively, on the singlet-triplet energy gap $\left(\Delta E_{\mathrm{ST}}\right)$. Smaller $\Delta E_{\mathrm{ST}}$ values allow more efficient rISC to convert triplet excitons into emissive singlet states. Efficient rISC can then out-compete nonradiative decay and other quenching processes. ${ }^{10-12}$ The rISC mechanism in these TADF emitters is a second-order spinvibronic coupling between a local-excited triplet state $\left({ }^{3} \mathrm{LE}\right)$ and a charge-transfer triplet state $\left({ }^{3} \mathrm{CT}\right)$ which mediates spin-orbit coupling to the $\mathrm{CT}$ singlet state $\left({ }^{1} \mathrm{CT}\right)$ leading to emission. ${ }^{13,14}$ In this case the ${ }^{3} \mathrm{LE}$ state acts as a mediator state for the spin flip.
It has been shown that the covalently linked donor (D) and acceptor (A) units with near-perpendicular D-A dihedral angles have strong molecular CT excited states and a small $\Delta E_{\mathrm{ST}}$ and have well separated highest occupied molecular orbital (HOMO)/lowest unoccupied molecular orbital (LUMO). ${ }^{15,16}$ However, a precise $90^{\circ}$ dihedral angle between the donor (D) and the acceptor (A) reduces the (emission) transition dipole moment and slows the fluorescence rate from the CT state, often leading to reduced emission efficiencies as quenching processes compete. ${ }^{14,17}$ Therefore, the $\mathrm{D}-\mathrm{A}$ angle needs to be optimized by selecting the correct molecular structure and degree of CT character and the host environment in order to achieve efficient TADF.

The molecular emitter design as well as the choice of the host play a key role in achieving efficient blue TADF OLEDs. The ideal host for blue materials needs to have a high triplet level (so as not to quench the high triplet emitter) as well as favorable

Received: April 11, 2019

Accepted: July 1, 2019

Published: July 17, 2019 


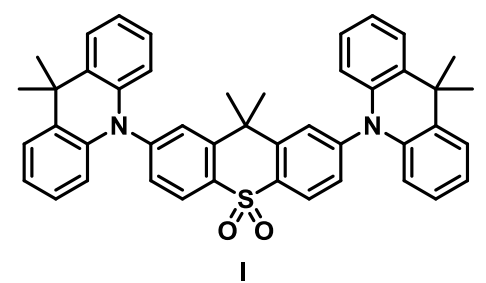<smiles>CC1(C)c2ccccc2N(c2ccc3c(c2)S(=O)(=O)c2cc(N4c5ccccc5C(C)(C)c5ccccc54)ccc2C3(C)C)c2ccccc21</smiles>

II<smiles>Cc1cc2c(cc1N1c3ccccc3C(C)(C)c3ccccc31)C(C)(C)c1cc(N3c4ccccc4C(C)(C)c4ccccc43)c(C)cc1S2(=O)=O</smiles>

III

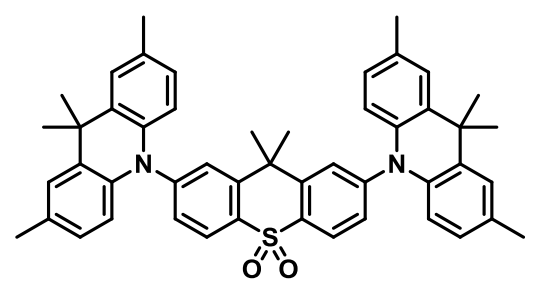

IV

Figure 1. Molecular structures studied in the present work.

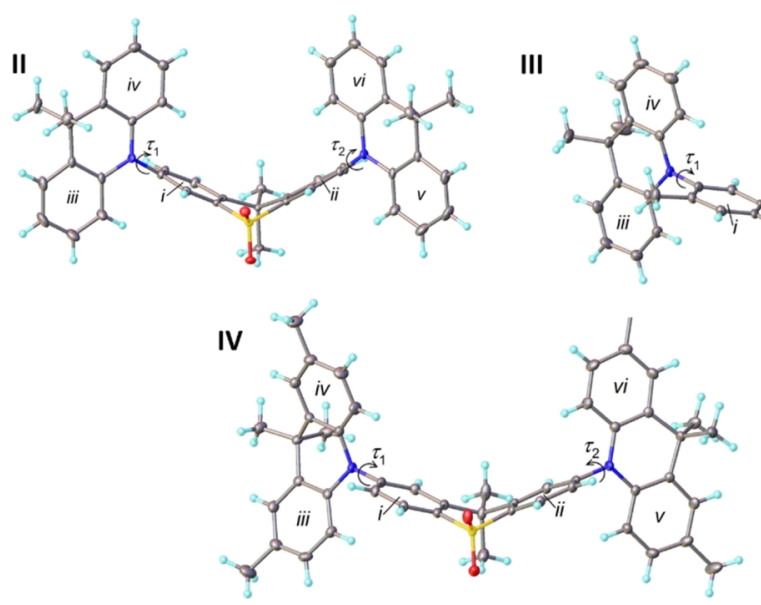

Figure 2. X-ray molecular structures of molecules II and III and IV.

carrier injection and transporting properties. ${ }^{18}$ The host also needs to provide an environment of suitable polarity, relaxing the energy of the ${ }^{1} \mathrm{CT}$ state toward that of the polarityindependent ${ }^{3} \mathrm{LE}$ state and thus minimizing the energy barrier to rISC. ${ }^{19}$ In this way, the rISC rate $\left(k_{\text {rISC }}\right)$ is optimized, and improved TADF and triplet harvesting can be achieved. ${ }^{2}$

Despite the currently known TADF emitter optimization strategies, significant challenges with blue emitters remain because of the high energy of emission and extensive device efficiency roll-off at high brightness. It is also highly challenging to simultaneously optimize all of the required parameters for an efficient OLED device. For example, redesigning the emitter to alter the average $\mathrm{D}-\mathrm{A}$ dihedral angle to optimize rISC often shifts the emission color undesirably. Herein, we report three systematic structural modifications of the D-A-D molecule 2,7-bis(9,9-dimethylacridin-10-yl)-9,9-dimethylthioxanthene$S, S$-dioxide (DDMA-TXO2) to investigate the structureproperty relationships relevant to blue TADF emitters. Donor (D) and acceptor (A) strength, structural isomerism, and sterically-induced D-A dihedral angle modifications are all investigated in detail in comparison with DDMA-TXO2, compound I (Figure 1). ${ }^{20}$ All these modifications were performed with an aim of developing efficient deep-blue emitters that improve upon the performance of the benchmark material I. Previously reported devices based on this material had a maximum external quantum efficiency (EQE) of $22.4 \%$ in the bis[2-(diphenylphosphino)phenyl] ether oxide (DPEPO) host and Commission Internationale de l'Éclairage (CIE) chromaticity coordinates of $(0.16,0.24) .{ }^{20}$ An independent study obtained a maximum EQE of $19.4 \%$ at ca. $100 \mathrm{~cd} \mathrm{~m}^{-2}$ with CIE coordinates of $(0.15,0.13)$ using a more complex device structure with a mixed host (UGH3:TSPO1) emitter layer. ${ }^{21}$ These studies further expose the very delicate balance between color purity and high EQE values.

The structures of the benchmark emitter I and the new variants designed and synthesized for this study (II-IV) are shown in Figure 1. The new molecules have been thoroughly characterized to confirm their structure and purity with ${ }^{1} \mathrm{H}$ and ${ }^{13} \mathrm{C}\left\{{ }^{1} \mathrm{H}\right\}$ nuclear magnetic resonance (NMR) spectroscopies, mass spectrometry, X-ray crystallography, and elemental analysis. Synthetic procedures and characterization data are included in the Supporting Information. New material II is a regioisomer of I with meta- rather than para-connectivity of the donor unit with respect to the acceptor sulfone functionality. Materials III and IV have modified D or A strength; in III, the strength of the A unit is decreased by the addition of methyl substituents. The rationale for methyl groups in III was to significantly modify the average $\mathrm{D}-\mathrm{A}$ torsion angle and to blueshift the emission. The strength of the donor in IV is increased because of the inductive effect of methyl substitution on the donor unit. 
In addition to its electron-donating effects, it has also been shown that the methyl groups can have a profound influence on the properties of molecules through steric effects. Stericallysubstituted $\mathrm{D}-\mathrm{A}-\mathrm{D}$ molecules with substituents on the donor often favor different conformers compared to the unsubstituted analogs leading to different HOMO/LUMO levels, which are critical to electrical device performance. ${ }^{22,23}$ Substitution of the donor in sterically-hindering positions was avoided in the present work to prevent the formation of undesirable axial conformers, which are detrimental to TADF performance. ${ }^{24}$ In the case of III, the structure was also anticipated to be more twisted on average due to the presence of the methyl substituents on the 9,9-dimethyl-thioxanthene-S,S-dioxide acceptor unit.

\section{RESULTS AND DISCUSSION}

X-ray Crystallography. Molecular structures from X-ray diffraction of single crystals of II, III, and IV are shown in Figure 2. Significant efforts to obtain a crystal structure of I did not yield crystals of X-ray quality: Large specimens obtained by controlled sublimation gave diffraction patterns indicative of two-dimensional order only, however, I is anticipated to have similar internal bond angles as IV. The thioxanthene (A) moiety in II, III, and IV is folded along the $\mathrm{S} \cdots \mathrm{C}\left(\mathrm{Me}_{2}\right)$ vector; the angle $(\theta)$ between the planes of its two arene rings varies widely as given in Table 1 . The acridine (D) moieties are also bent along the $\mathrm{N} \cdots$

Table 1. Interplanar Angles (deg) Obtained Using X-ray Crystallography ${ }^{a}$

\begin{tabular}{lllr} 
& \multicolumn{1}{c}{ II } & \multicolumn{1}{c}{ IIIa $/ \mathbf{b}^{b}$} & IV \\
$\theta$ & 126.6 & $151.4 / 152.8$ & 139.9 \\
$\omega_{1}$ & 3.6 & $5.9 / 7.8$ & 30.4 \\
$\omega_{2}$ & $(3.6)$ & $5.2 / 5.8$ & 4.4 \\
$\tau_{1}$ & 81.3 & $83.5 / 84.6$ & 82.2 \\
$\tau_{2}$ & $(81.3)$ & $87.0 / 88.6$ & 83.6
\end{tabular}

${ }^{a}$ Experimental details available in the Supporting Information. ${ }^{b}$ Different solvate details in the Supporting Information.

$\mathrm{C}\left(\mathrm{Me}_{2}\right)$ vectors; the bending, as defined by arene/arene interplanar angles $\left(\omega_{1,2}\right)$, is slight, except for one acridine in IV. This picture agrees with the literature: of the eight reported 9,9-dimethylacridine structures with a $\mathrm{C}$-substituent at $\mathrm{N}$, three have $\omega$ of $31^{\circ}-34^{\circ}$ and five of $1^{\circ}-11^{\circ}$. In each case, the twist $\left(\tau_{1,2}\right)$ around the $\mathrm{C}($ thioxanthene $)-\mathrm{N}$ bond is nearly perpendicular $\left(81^{\circ}-89^{\circ}\right)$ and precludes any $\pi$-conjugation. III shows a slightly more perpendicular structure compared to other reported molecules. However, as previously discussed, a considerable variation in $\theta$ is observed for III when compared to the X-ray structures of II and IV and must be due in part to the methyl substituent on each acceptor arene ring. It appears that the meta-methyl groups alter the degree of planarization of the acceptor. Initially it was expected that the methyl groups in molecule III will have the greatest effect on the dihedral angle $\tau$ (making III more orthogonal); however, this may not be the case. It is important to stress that the average $\mathrm{D}-\mathrm{A}$ torsion angle in an amorphous film might not be represented by X-ray crystal data. Effects observed could be due to crystal structure packing and are not necessarily applicable in the films relevant to optical measurements and devices. The X-ray structure does show that the molecules can adopt a perpendicular D-A conformation, which is beneficial for TADF emission. Considering these factors, the X-ray structure of III does still suggest that the methyl substituents would shift the D-A torsion angle more to the perpendicular on average, which was a pivotal part of the rational molecular design of III. The X-ray analysis reveals no axial conformers in these $\mathrm{D}-\mathrm{A}-\mathrm{D}$ molecules. This was expected for the more rigid and planar acridine donor unit, compared to the more flexible and significantly folded phenothiazine unit. ${ }^{24}$

Steady-State and Time-Resolved Spectroscopy. The absorption spectra of the new molecules II-IV as well as the reference emitter I are shown in Figure 3. The weak absorption

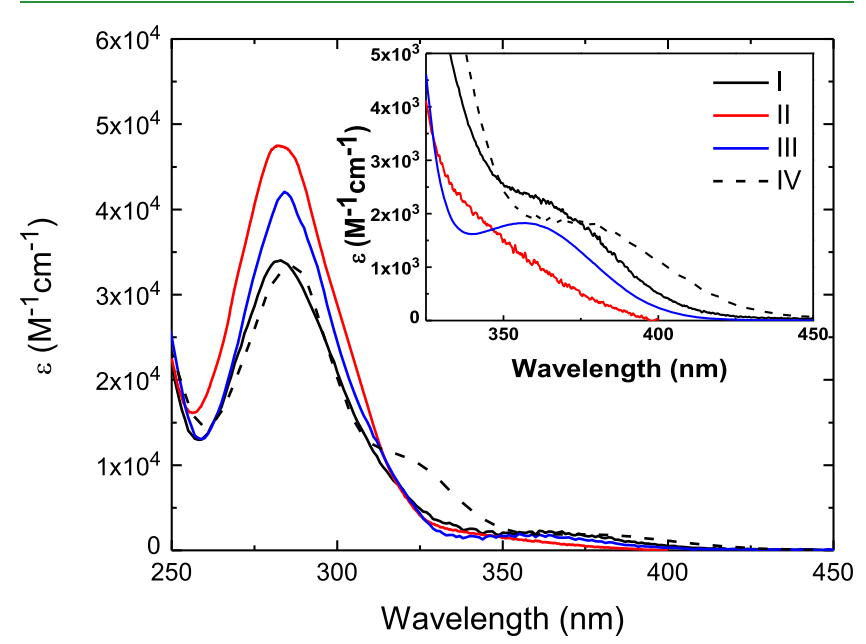

Figure 3. Absorption spectra of I-IV in DCM solvent; the inset graph is an expansion of the $325-450 \mathrm{~nm}$ region.

feature observed for I and IV at around $360 \mathrm{~nm}$ does not appear in the spectra of either of the individual D or the A units and has therefore been assigned to the "direct" CT absorption, an $\mathrm{n}-\pi^{*}$ $\left(\sigma-\pi^{*}\right)$ [or mixed $\left.\pi \pi^{*} / \mathrm{n} \pi^{*}\left(\pi \pi^{*} / \sigma \pi^{*}\right)\right]$ transition in the DA-D molecules. ${ }^{20,25}$ Molar absorption coefficients $\left(\varepsilon_{\mathrm{MAX}}\right)$ of these molecules at $360 \mathrm{~nm}$ are very low as is expected of an $n-\pi^{*}$ transition, ranging between ca. 1500 and $2500 \mathrm{M}^{-1} \mathrm{~cm}^{-1}$ in dichloromethane (DCM) (Table 2). In line with our previous investigations, the transition at around $280-290 \mathrm{~nm}$ can be assigned to local excitation of the D unit. ${ }^{20}$ Material IV exhibits a shoulder at $325 \mathrm{~nm}$ that is not present in the other molecules, however, this has been shown to arise from its individual $\mathrm{D}$ unit (Figure S2). This indicates that the methyl groups substituted on the $\mathrm{D}$ unit clearly affect the electronic structure of the donor.

Table 2. Steady-State Photophysical Properties of the Target Compounds. $\lambda_{\text {MAX }}, \varepsilon_{\text {MAX }}(285 / 360 \mathrm{~nm}), \lambda_{\text {FLU }}$, and Steady State Were Measured in DCM Solution, whereas PLQYs $\left(\Phi_{\mathrm{F}}\right)$ Were Measured in Small Molecule Host (DPEPO) under a Nitrogen Atmosphere

\begin{tabular}{|c|c|c|c|c|}
\hline & I & II & III & IV \\
\hline$\lambda_{\mathrm{MAX}} / \mathrm{nm}^{a}$ & 365 & 282 & 359 & 370 \\
\hline$\varepsilon_{\mathrm{MAX}} / \mathrm{M}^{-1} \mathrm{~cm}^{-1 b}$ & $\begin{array}{c}33558 / \\
1798\end{array}$ & $\begin{array}{c}48477 / \\
2490\end{array}$ & $\begin{array}{c}41864 / \\
1576\end{array}$ & $\begin{array}{c}33986 / \\
1905\end{array}$ \\
\hline$\lambda_{\mathrm{FLU}} / \mathrm{nm}^{c}$ & 504 & 506 & 495 & 538 \\
\hline $\mathrm{SS} / \mathrm{cm}^{-1 d}$ & 7556 & 15698 & 7653 & 8440 \\
\hline$\Phi_{\mathrm{F}}^{e}$ & $80 \%$ & $62 \%$ & $88 \%$ & $73 \%$ \\
\hline
\end{tabular}

${ }^{a}$ Absorption peak maximum at RT. ${ }^{b}$ Molar emission coefficient at $285 \mathrm{~nm} / 360 \mathrm{~nm}$. ${ }^{c}$ Emission peak maximum at RT. ${ }^{d}$ Stokes shift. ${ }^{e}$ Absolute emission quantum yield $\left(\Phi_{\mathrm{F}} \pm 10 \%\right)$ using $330 \mathrm{~nm}$ excitation, all measured using $25 \mathrm{vol} \%$ evaporated samples in DPEPO with the exception of IV which was drop-cast at $10 \mathrm{wt} \%$. 

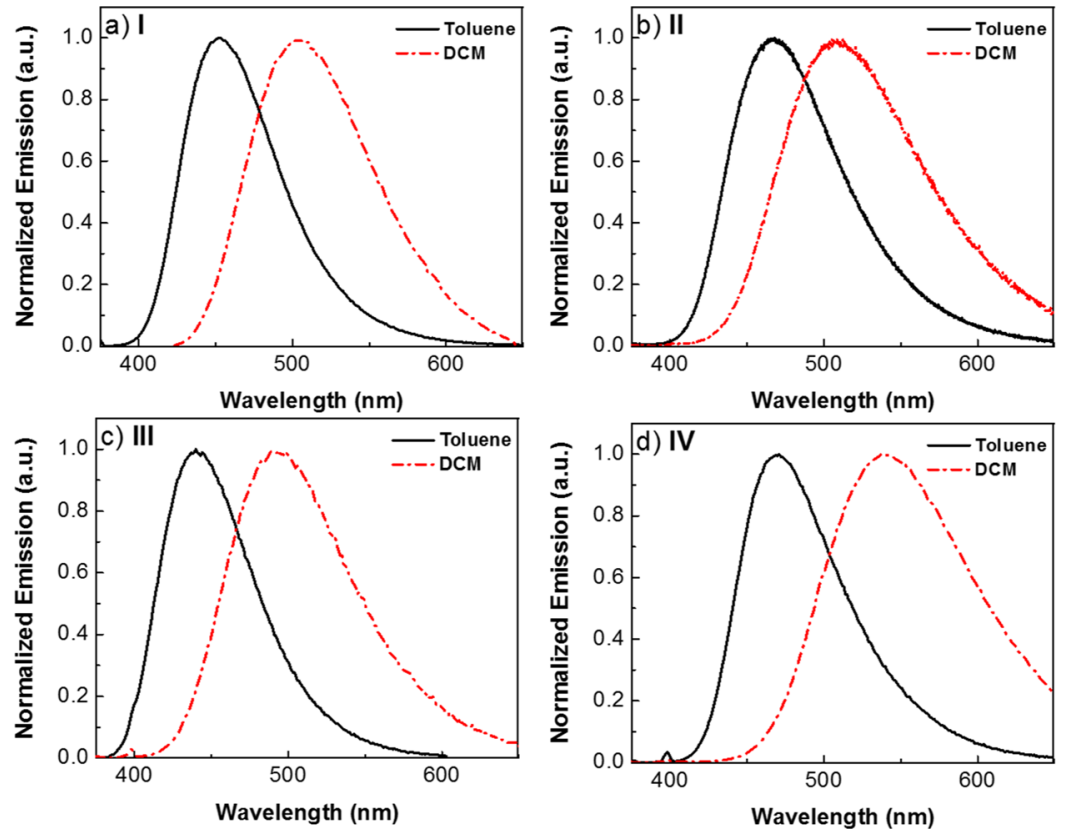

Figure 4. Normalized PL spectra of (a) I, (b) II, (c) III, and (d) IV in toluene and DCM solvent; excitation wavelength was $355 \mathrm{~nm}$.
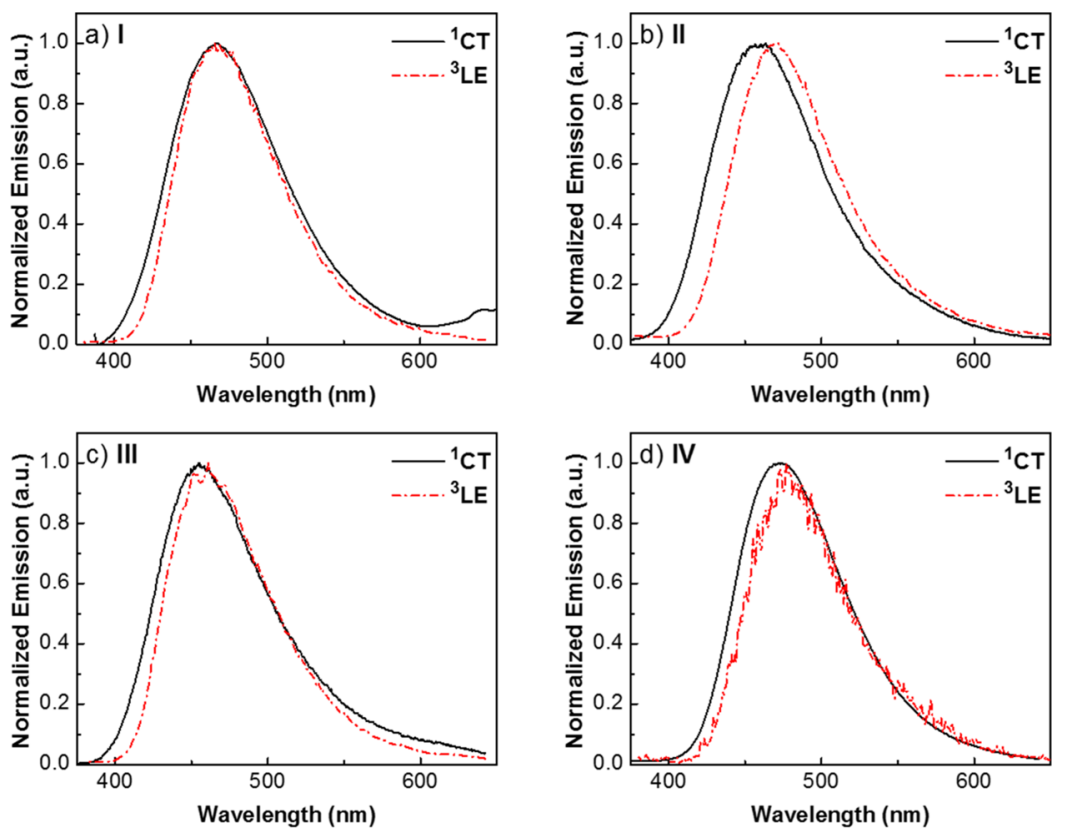

Figure 5. PL (at RT) and phosphorescence (recorded at $80 \mathrm{~K}$ with $70 \mathrm{~ms}$ delay-time) spectra of (a) I, (b) II, (c) III, and (d) IV in DPEPO.

It is expected that through the increased $\pi$ density in acridine (inductive electron donation from the methyl groups), the lone pair electrons of the donor $\mathrm{N}$ are less delocalized into the acridine arene rings, giving rise to a more pronounced $n \pi^{*}$ transition of the $\mathrm{D}$ unit. This band is clearly absent in all donor unsubstituted molecules I-III.

The emission spectra of all molecules exhibit strong positive solvatochromism and a Gaussian emission profile, consistent with the CT excited states, as in other D-A-D molecules. ${ }^{26}$ The Gaussian emission has been observed in low polarity toluene $(\varepsilon=2.43)$ as well as significantly more polar DCM $(\varepsilon=$ 8.93 ), indicating strong CT character present in these molecules (Figure 4). The emission maxima in DCM are approximately the same for all molecules, except for IV which shows a considerably red-shifted emission spectrum. All molecules exhibit large Stokes shifts typical of the CT states in TADF materials, Table 2. ${ }^{27}$ The Stokes shift of II appears much greater than those for the other molecules because it is estimated using the $\pi \pi^{*}$ transition and not the $\mathrm{n} \pi^{*}$ transition used for the other molecules. This is because the $\mathrm{n} \pi^{*}$ transition for II is blueshifted and overlaps with the $\pi \pi^{*}$ transition, and hence the peak of the $\mathrm{n} \pi^{*}$ transition is not experimentally accessible. Molecule II also has the largest $\pi \pi^{*}$ extinction coefficient, indicating less delocalization of the acridine $\pi$ density into A. The quantum yields are high (62-88\%) for all I-IV.

Time-resolved emission decay measurements were performed as previously described ${ }^{28,29}$ and allow quantification of the prompt and delayed emission lifetimes $\left(\tau_{\mathrm{PF}}\right.$ and $\tau_{\mathrm{DF}}$, 
respectively) and reveal the rISC rates for each material $\left(k_{\mathrm{rISC}}\right)$. For these time-resolved experiments, the materials were dispersed at $10 \mathrm{wt} \%$ in DPEPO in anticipation of OLED device fabrication in this host. The DPEPO host was selected because of the scarcity of other suitable OLED host materials with sufficiently high triplet energy and acceptable chargetransport properties. Previous work on molecule I shows that measurements in the less polar Zeonex host resulted in a larger singlet-triplet gap and lower photoluminescence quantum yield (PLQY) compared to the DPEPO host. ${ }^{20}$ Zeonex is a useful medium for fundamental photophysical studies, however substantial changes in photophysical properties are observed between Zeonex and DPEPO. Therefore, detailed photophysics in a suitable OLED host of choice (DPEPO) was performed so that direct comparison between photophysical and electroluminescence (EL) data could be made. The photoluminescence (PL) spectra in DPEPO (Figure 5) have comparable onset energies to those in toluene (Figure 4). This is consistent with the solid-state environment being of similar polarity with that of toluene. ${ }^{17}$ In the case of IV, its red-shifted emission compared to I reveals the increased donor strength. The paramethyl substituents of IV are unlikely to exhibit any steric effects because of their remote position of substitution.

The delayed lifetimes of all molecules in DPEPO at room temperature (RT) (Table 3 and Figure 6) are similar (ca. $3 \mu$ s),

Table 3. Summary of Energy Levels and Kinetic Parameters from Time-Resolved Emission of the Target Compounds in the DPEPO Matrix

$\begin{array}{lcccc} & \text { I } & \text { II } & \text { III } & \text { IV } \\ { }^{3} \mathrm{LE} / \mathrm{eV}^{a} & 2.98 & 2.98 & 3.02 & 2.91 \\ { }^{1} \mathrm{CT} / \mathrm{eV}^{b} & 3.04 & 3.11 & 3.07 & 2.97 \\ \Delta E_{\mathrm{ST}} / \mathrm{eV}^{c} & 0.06 & 0.13 & 0.05 & 0.06 \\ \tau_{\mathrm{PF}} / \mathrm{ns}^{d} & 40 & 83.5 & 47 & 41.4 \\ \tau_{\mathrm{DF}} / \mu \mathrm{s}^{e} & 4.5 & 8.1 & 3 & 3.7 \\ k_{\mathrm{rISC}} / \times 10^{6} \mathrm{~s}^{-1 f} & 1.2 & 0.38 & 2.8 & 1.1\end{array}$

${ }^{a}$ Determined from the onset of the phosphorescence emission at $20 \mathrm{~K}$ (or $80 \mathrm{~K}$ for IV) with greater than $70 \mathrm{~ms}$ delay-time. ${ }^{b}$ Determined using the onsets of the assigned $\mathrm{CT}$ emission band. ${ }^{c}$ Determined using the onsets of ${ }^{3} \mathrm{LE}$ and ${ }^{1} \mathrm{CT}$. ${ }^{d}$ Prompt fluorescence lifetime. ${ }^{e}$ Delayed fluorescence lifetime. ${ }^{f_{\text {rISC }}}$ rate determined using previously reported methods. ${ }^{30}$

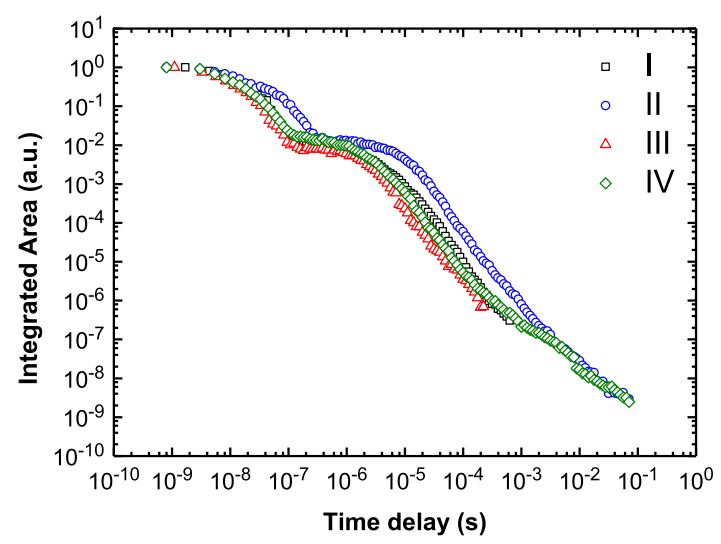

Figure 6. Time-resolved emission decay for I-IV in the DPEPO matrix; the curves were obtained using $355 \mathrm{~nm}$ excitation wavelength.

with the exception of II which showed a reduced rISC rate and an extended delayed lifetime. In order to estimate the rISC rates, the fitting method previously described by Haase and coworkers was used. ${ }^{30}$ It was found that $\mathrm{k}_{\mathrm{rISC}}$ follows the order III > $\mathbf{I} \approx \mathbf{I V}>$ II (Table 3 ) in excellent agreement with the measured $\Delta E_{\mathrm{ST}}$. As material IV was found to have almost identical properties to I, a lower PLQY, and red-shifted emission, IV was discounted from further investigation in devices. Triplet-triplet annihilation has been excluded as the cause of the delayed emission by means of laser power dependence measurements; linear dependence with a slope of 1 was established for molecules I-IV and most importantly for III (Figure S3). ${ }^{31}$

The temperature dependence of the emission in the solid state for all four molecules was measured in DPEPO (Figure 7). The data for $\mathbf{I}$ is consistent with previous report. ${ }^{20}$

Time-dependent emission decays were collected between 80 and $290 \mathrm{~K}$ and with suitable time delays (TDs) to unambiguously capture the CT singlet and phosphorescence emission (Figure 7) to unambiguously capture the CT singlet and phosphorescence emission (Figure 5). The phosphorescence of III (TD = 70 ms) has an onset value of $3.02 \pm 0.02$ $\mathrm{eV}$; this is the highest measured energy of the localized triplet $\left({ }^{3} \mathrm{LE}\right)$ in the series of molecules presented here. In contrast, IV has the lowest lying ${ }^{3} \mathrm{LE}$ state with an onset energy of $2.91 \pm 0.02$ $\mathrm{eV}$ in DPEPO. The ${ }^{3} \mathrm{LE}$ of I and II appear to be isoenergetic. The ${ }^{3} \mathrm{LE}$ emission spectra of all four molecules of this series appear to be a mix of ${ }^{3} \mathrm{LE}$ of the $\mathrm{D}$ and $\mathrm{A}$ units. The phosphorescence emission has been assigned as ${ }^{3} \mathrm{LE}$ despite the broadness of the emission due to the $\mathrm{C}-\mathrm{N}$ donor-acceptor bridge in molecules I-IV, and the conjugation across this bond is very limited due to the near-perpendicular D-A orientation. Therefore, some of the local character of these isolated donor units remains in the D$\mathrm{A}-\mathrm{D}$ system. These localized orbitals are the reason why $\Delta E_{\mathrm{ST}}$ is so small in these compounds as the localized triplet state is located predominantly on the $\mathrm{D}$ or A because of lack of conjugation, whereas the CT state is likely distributed across the whole molecule. This local triplet character has previously been observed in several similar systems. ${ }^{20,32}$

At short delays (i.e., prompt emission), broad Gaussian bands characteristic of CT emission are observed. ${ }^{33}{ }^{1} \mathrm{CT}$ onset energies ranging from $2.97 \pm 0.02 \mathrm{eV}$ in IV to $3.11 \pm 0.02 \mathrm{eV}$ for II are reported. There is a small time-dependent apparent red shift of the ${ }^{1} \mathrm{CT}$ emission over the first $100 \mathrm{~ns}$ as exemplified for III in Figure S4. This red shift is similar to what has been previously observed in I because of dihedral angle heterogeneity. ${ }^{17,20,34}$ From Figure S4, it is clear that only the blue edge of the emission band red shifts during the first $15 \mathrm{~ns}$, and the whole band does not red shift, clearly showing the faster decay of the bluest states, that is, those with the smallest dihedral angle. For III, there is no loss of DF at $160 \mathrm{~K}$ as compared to $290 \mathrm{~K}$; this indicates a very small activation energy $\left(E_{\mathrm{A}}\right) . E_{\mathrm{A}}$ also appears to be small for IV as indicated by negligible differences in the DF intensity between 290 and $160 \mathrm{~K}$. The PF of III $\left(\tau_{\mathrm{PF}}=47 \mathrm{~ns}\right)$ is slower than that of $\mathbf{I}\left(\tau_{\mathrm{PF}}=40 \mathrm{~ns}\right)$ because of a more perpendicular $\mathrm{D}-\mathrm{A}$ conformation enforced by the methyl groups appended to the acceptor of III. Compound II is characterized by the slowest emission in this series caused by the largest $\Delta E_{\mathrm{ST}}$ : consequently both ISC and rISC processes are slow leading to extended prompt and delayed lifetimes.

Comparing the onsets of both the prompt ${ }^{1} \mathrm{CT}$ emission and the ${ }^{3} \mathrm{LE}$ emission from Figure 5 allows $\Delta E_{\mathrm{ST}}$ to be estimated, as reported in Table 3. $\Delta E_{\mathrm{ST}}$ of $I$ in DPEPO is $60 \mathrm{meV}$ with a rISC rate of $1.2 \times 10^{6} \mathrm{~s}^{-1}$. In contrast, II has $\Delta E_{\mathrm{ST}}$ of $130 \mathrm{meV}$ and a significantly slower rISC rate of $0.38 \times 10^{6} \mathrm{~s}^{-1}$, which also correlates with its lower PLQY. Finally, III has $\Delta E_{S T}$ of $50 \mathrm{meV}$ 

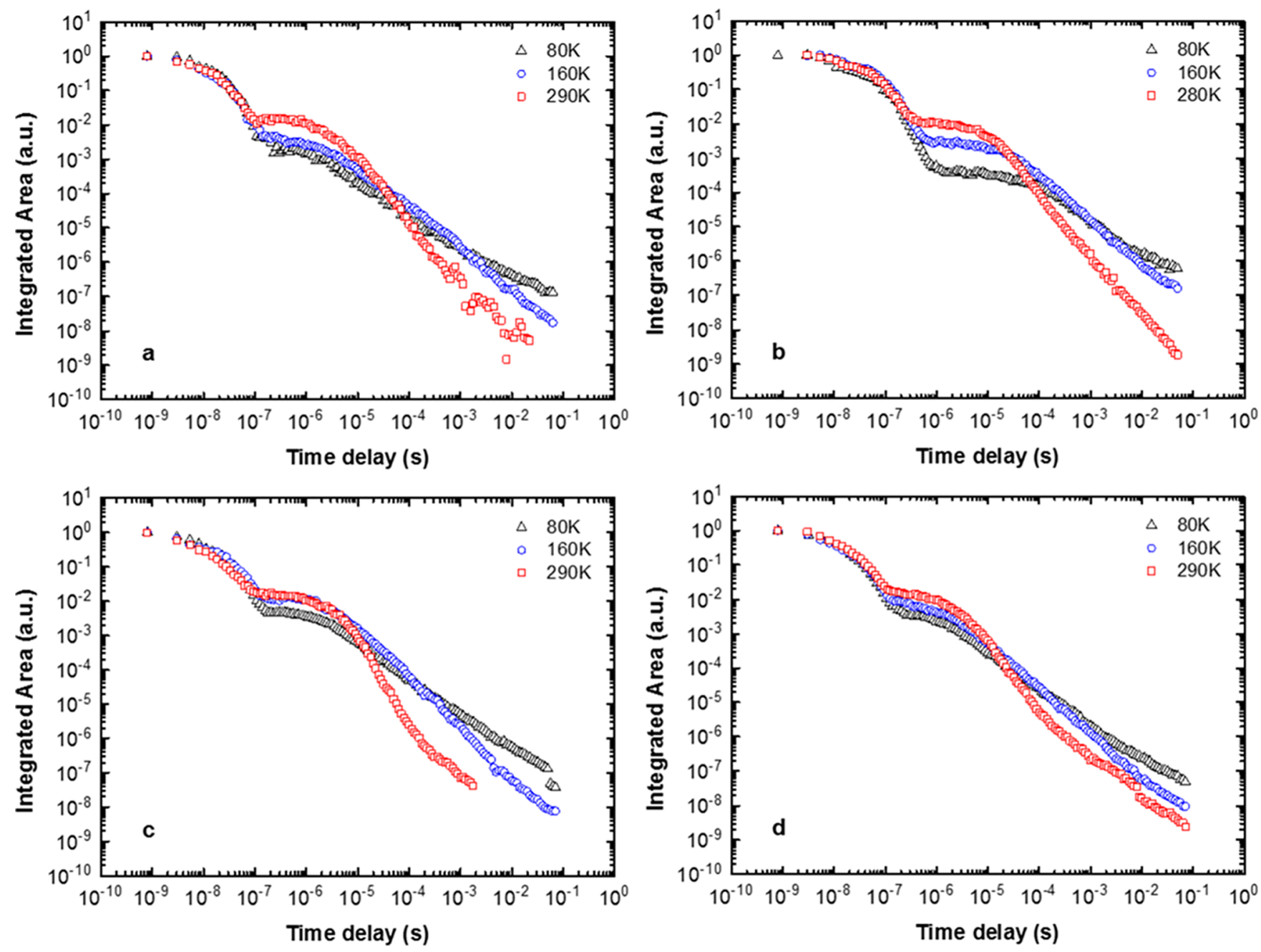

Figure 7. Time-resolved fluorescence decay of (a) I, (b) II, (c) III, and (d) IV in the DPEPO matrix.

Table 4. Electrical Properties of Devices Based on I, II, and III Including EQE $\left(\boldsymbol{\eta}_{\text {Ext }}\right)$, Luminance $(L)$, Current Efficiency $\left(\boldsymbol{\eta}_{\mathrm{L}}\right)$, Luminous Efficiency $\left(\boldsymbol{\eta}_{\mathrm{P}}\right)$, CIE Coordinates at Maximum Value, and fwhm.

\begin{tabular}{lcccccccc} 
& $L^{a}\left(\mathrm{~cd} / \mathrm{m}^{2}\right)$ & $\eta_{\text {ext }}{ }^{a}(\%)$ & $\eta_{\mathrm{L}}{ }^{a}(\mathrm{~cd} / \mathrm{A})$ & $\eta_{\mathrm{P}}{ }^{a}(\mathrm{~lm} / \mathrm{W})$ & $\eta_{\mathrm{ext}}{ }^{b}(\%)$ & $\eta_{\mathrm{ext}}{ }^{c}(\%)$ & $\mathrm{CIE}^{d}(x, y)$ & $\mathrm{fwhm}(\mathrm{nm})$ \\
\hline I & 4204 & 21.2 & 36.8 & 27.5 & 20 & 19.3 & $0.16,0.25$ & 81 \\
II & 1949 & 15.6 & 29.6 & 18.6 & 14.3 & 11.2 & $0.17,0.29$ & 93 \\
III & 2765 & 22.6 & 31.7 & 24.3 & 22.5 & 19.9 & $0.15,0.18$
\end{tabular}

${ }^{a}$ Measured maximum values. ${ }^{b}$ Measured values at a luminance of $100 \mathrm{~cd} \mathrm{~m}^{-2} .{ }^{c}$ Measured values at a luminance of $1000 \mathrm{~cd} \mathrm{~m}^{-2}$. ${ }^{d}$ Measured values at maximum $\eta_{\text {ext }}$.

and the fastest rISC rate recorded in this work at $2.8 \times 10^{6} \mathrm{~s}^{-1}$. The energy gap between ${ }^{3} \mathrm{LE}$ and ${ }^{1} \mathrm{CT}$ can be tuned by host polarity, and DPEPO was identified as a suitable host for the bluest material in the series based on the triplet energy. ${ }^{35}$ The DPEPO triplet energy is $3.05 \pm 0.02 \mathrm{eV}$ measured in a neat film at $80 \mathrm{~K}$ using $266 \mathrm{~nm}$ excitation wavelength. ${ }^{20}$

Devices. To evaluate I, II, and III in devices, the materials were purified by sublimation and used in the following general device structure: ITO/NPB (40 nm)/TSBPA $(10 \mathrm{~nm}) /$ emitter I-IV:DPEPO $(30 \mathrm{~nm}) / \mathrm{DPEPO}(10 \mathrm{~nm}) / \mathrm{TPBi}(40 \mathrm{~nm}) / \mathrm{LiF} /$ $\mathrm{Al}$ (Figure S5). The emission layer was optimized at 35\% dopant concentration for I-III in DPEPO; NPB is $N, N^{\prime}$-bis(naphthalene-1-yl)-N,N'-bis(phenyl)benzidine, ITO is indium tin oxide, TSBPA is 4,4-(diphenylsilanediyl)bis $(N, N$-diphenylaniline), and TPBi is 1,3,5-tris( $N$-phenylbenzimidazol-2-yl)benzene. An additional layer of DPEPO is used as a hole- blocking layer and to prevent TPBi (with a low lying ${ }^{3} \mathrm{LE}$ ) from quenching the blue emitter at the host material interface. Importantly, the use of TSBPA as a hole-transport layer effectively prevents the formation of the green electromer of 4,4-(diphenylmethylene) bis $(N, N$-diphenylamine) previously attributed to an exciplex formation between TPBi and $\mathbf{I}^{20}$ The HOMO levels of I-IV were estimated from the cyclic voltammetry data are very similar (the LUMO levels were estimated from the measured HOMO and the band gap using absorption spectra) (Figure S1); hence, the electrical properties of the devices produced are expected to be similar (Table S2). All device performance characteristics are reported (Table 4 and Figure 8). The optimized high dopant concentration (35\%) of I-III in DPEPO was chosen after screening concentrations of $15-50 \%$ and is consistent with the optimized 30\% of DDMA$\mathrm{TXO} 2$, compound $\mathrm{I}$, in a mixed host reported by Lee and Lee. ${ }^{21}$ 

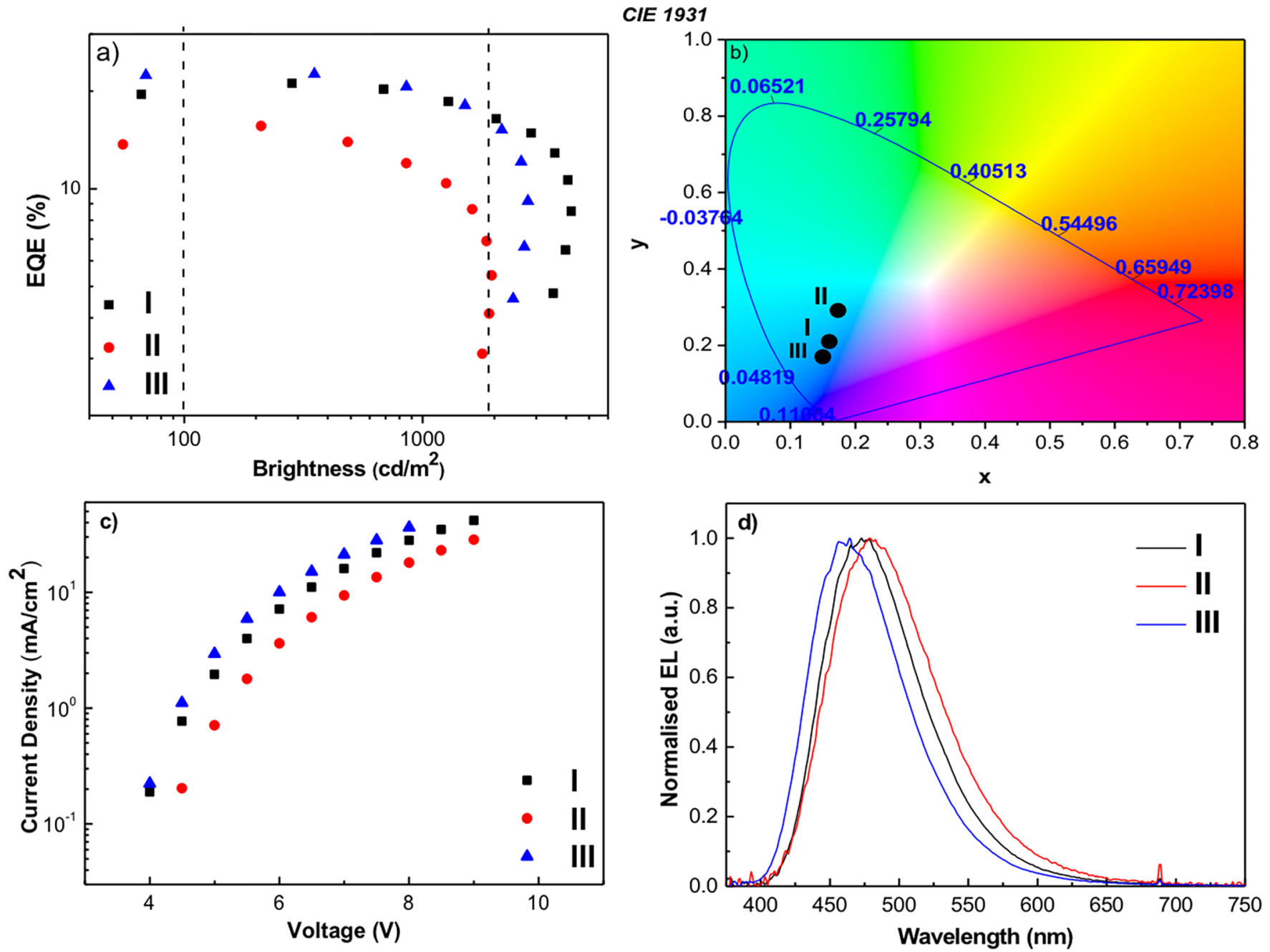

Figure 8. (a) EQE vs brightness, (b) CIE color coordinates, (c) current density vs voltage, and (d) EL spectra of I-III and device architecture described above.

It has been shown that the hole transport from the TADF material is a reason for needing such high concentrations of TADF material, as DPEPO does not transport holes, and so the TADF emitter must also perform this role. ${ }^{36,37}$

The same device architecture was employed for I-III to enable a meaningful comparison within the whole series, rather than comparing with previously reported devices of $\mathbf{I}$, which used different architectures. ${ }^{20,21}$

For all devices, the maximum EQEs achieved were in agreement with the measured PLQYs (approximated using $\mathrm{EQE}=\mathrm{PLQY} \times$ charge balance $\times$ outcoupling $=0.25 \times \mathrm{PLQY})$, assuming there is no horizontal molecular alignment. ${ }^{38}$ The best performance was observed for material III with a maximum EQE of $22.6 \%$ with CIE coordinates $(0.15,0.18)$ and full width at half maximum (fwhm) of $78 \mathrm{~nm}$. The EL peak is at $461 \mathrm{~nm}$ (Figure 8d) and does not shift with voltage where the maximum intensity is achieved (Figure S6). The OLED device for III maintains $88 \%$ of its maximum EQE at $1000 \mathrm{~cd} \mathrm{~m}^{-2}$. In comparison to the reference material I when using the same device structure, an improvement in efficiency and deeper blue color is observed for III. The roll off of III is comparable to that of I (device I maintains $91 \%$ of its maximum EQE at $1000 \mathrm{~cd}$ $\mathrm{m}^{-2}$ ). Importantly, we have demonstrated that the EQE of III is higher than that of $\mathrm{I}$ at 100 and $1000 \mathrm{~cd} \mathrm{~m}^{-2}$ with color coordinates more desirable for blue OLED applications (Figure 8a). In contrast, consistent with its larger $\Delta E_{\mathrm{ST}}$, slower rISC, and lower PLQY, the device performance of II was not as high as I or III $\left(\mathrm{EQE}_{\max }\right.$ for II of $\left.14.1 \%\right)$. We believe that the increase in EQE for compound III is due to its high rISC rate and fast $\tau_{\mathrm{DF}}$. The prompt fluorescence rate is so much faster than the rISC (ns vs $\mu \mathrm{s}$ ), and therefore, small changes in the CT character and $\tau_{\mathrm{PF}}$ will not significantly affect the devices; however, the faster rISC rate allows for efficient triplet harvesting. Emission in TADF molecules comprises both prompt $\mathrm{CT}$ and delayed $\mathrm{CT}$ emissions; thus identifying a single cause to changes in overall PLQY is complex in these systems.

The weaker acceptor of III causes the blue shift of the emission, which results in improved device blue color coordinates of III versus I. It is suggested that the average DA dihedral angle also contributes to III's superior optical and electrical performance by restricting the $\mathrm{D}-\mathrm{A}$ dihedral angle closer to $90^{\circ}$ in III. The ${ }^{1} \mathrm{CT}$ and ${ }^{3} \mathrm{LE}$ states are shifted to higher energies because of less electronic communication of the donor $\mathrm{N}$ lone pair with the acceptor. It is important to note that this effect, presumably due to steric interactions of the donor with the acceptor methyl substituents, raises both the CT and the triplet together, maintaining a low $\Delta E_{\mathrm{ST}}$ critical for efficient TADF.

These OLED data for III are very competitive with the recent efficient devices of TADF emitters with similar CIE coordinates. 
For example, D-A molecules recently published by Woo and co-workers gave an $\mathrm{EQE}_{\max }$ of $23.7 \%$ (CIE 0.148, 0.158) and $16 \%(0.148,0.131) .{ }^{39}$ However, although these devices are deeper-blue than III, and they exhibit considerable roll-off to 14.5 and $8.5 \%$ at $100 \mathrm{~cd} \mathrm{~m}^{-2}$.

\section{CONCLUSIONS}

Summarizing, a series of new blue TADF molecules has been rationally designed, synthesized, and characterized in detail in order to develop understanding of how to improve the TADF efficiency and create more efficient blue OLED devices. Detailed photophysical measurements successfully identify III as an improved version of $\mathbf{I}$ for blue emission, which is attributed to its sterically controlled $\mathrm{D}-\mathrm{A}$ angle. In IV, the addition of methyl groups on the donor slightly increases the donor strength and red shifts the emission. Interestingly, the methyl groups do not significantly alter the rISC rate. OLED devices fabricated with III have higher performance compared to reference I, with CIE color coordinates in the deeper blue region. Finally, it has been demonstrated that regioisomerism has a huge effect on the rISC rates and PLQY even though the ${ }^{1} \mathrm{CT}$ level is not changed significantly, as exemplified by comparison of I and II.

\section{ASSOCIATED CONTENT}

\section{S Supporting Information}

The Supporting Information is available free of charge on the ACS Publications website at DOI: 10.1021/acsami.9b06364.

Details of synthetic procedures, copies of NMR spectra, X-ray crystallographic data, solution electrochemical data, and additional photophysical and OLED data (PDF) (ZIP)

\section{AUTHOR INFORMATION}

\section{Corresponding Authors}

*E-mail: patrycja.stachelek@durham.ac.uk (P.S.).

*E-mail: a.p.monkman@durham.ac.uk (A.P.M.).

*E-mail: m.r.bryce@durham.ac.uk (M.R.B.).

\section{ORCID $\odot$}

Patrycja Stachelek: 0000-0003-3002-3986

Jonathan S. Ward: 0000-0002-4985-8572

Andrew Danos: 0000-0002-1752-8675

Marco Colella: 0000-0003-1627-2978

Andrei S. Batsanov: 0000-0002-4912-0981

Martin R. Bryce: 0000-0003-2097-7823

Andrew P. Monkman: 0000-0002-0784-8640

\section{Author Contributions}

P.S. and J.S.W. contributed equally. All authors have given approval to the final version of the manuscript.

\section{Notes}

The authors declare no competing financial interest.

\section{ACKNOWLEDGMENTS}

The authors would like to acknowledge funding for HyperOLED and EXCILIGHT projects from the European Union's Horizon 2020 research and innovation program under grant agreement numbers 732013 and 674990, respectively. J.S.W., M.R.B., and A.P.M. thank EPRSC grant EP/L02621X/1 for funding. P.L.d.S. thanks the CAPES Foundation, Ministry of Education of Brazil, Brasilia-DF 70040-020, Brazil, in particular, the Science Without Borders Program for a $\mathrm{PhD}$ studentship.

\section{REFERENCES}

(1) Dias, F. B.; Bourdakos, K. N.; Jankus, V.; Moss, K. C.; Kamtekar, K. T.; Bhalla, V.; Santos, J.; Bryce, M. R.; Monkman, A. P. Triplet Harvesting with $100 \%$ Efficiency by Way of Thermally Activated Delayed Fluorescence in Charge Transfer OLED Emitters. Adv. Mater. 2013, 25, 3707-3714.

(2) Bui, T.-T.; Goubard, F.; Ibrahim-Ouali, M.; Gigmes, D.; Dumur, F. Recent Advances on Organic Blue Thermally Activated Delayed Fluorescence (TADF) Emitters for Organic Light-Emitting Diodes (OLEDs). Beilstein J. Org. Chem. 2018, 14, 282-308.

(3) Xiao, P.; Dong, T.; Xie, J.; Luo, D.; Yuan, J.; Liu, B. Emergence of White Organic Light-Emitting Diodes Based on Thermally Activated Delayed Fluorescence. Appl. Sci. 2018, 8, 299-330.

(4) Uoyama, H.; Goushi, K.; Shizu, K.; Nomura, H.; Adachi, C. Highly Efficient Organic Light-Emitting Diodes from Delayed Fluorescence. Nature 2012, 492, 234-238.

(5) Wang, C.; Zheng, Y.-Q.; Tang, J.; Yu, J.-L.; Yang, F.; Wei, B.; Li, X.F.; Zhang, J.-H. Highly Efficient Green TADF Organic Light-Emitting Diodes by Simultaneously Manipulating Hole and Electron Transport. Nanotechnology 2019, 30, 115201-115209.

(6) dos Santos, P. L.; Ward, J. S.; Congrave, D. G.; Batsanov, A. S.; Eng, J.; Stacey, J. E.; Penfold, T. J.; Monkman, A. P.; Bryce, M. R. Triazatruxene: A Rigid Central Donor Unit for a D-A Thermally Activated Delayed Fluorescence Material Exhibiting Sub-Microsecond Reverse Intersystem Crossing and Unity Quantum Yield via Multiple Singlet-Triplet State Pairs. Adv. Sci. 2018, 5, 1700989-1700997.

(7) Huang, Z.; Xiang, S.; Zhang, Q.; Lv, X.; Ye, S.; Guo, R.; Wang, L. Highly Efficient Green Organic Light Emitting Diodes with Phenanthroimidazole-Based Thermally Activated Delayed Fluorescence Emitters. J. Mater. Chem. C 2018, 6, 2379-2386.

(8) Wong, M. Y.; Zysman-Colman, E. Purely Organic Thermally Activated Delayed Fluorescence Materials for Organic Light-Emitting Diodes. Adv. Mater. 2017, 29, 1605444-1605497.

(9) Cai, X.; Su, S.-J. Marching Toward Highly Efficient, Pure-Blue, and Stable Thermally Activated Delayed Fluorescent Organic LightEmitting Diodes. Adv. Funct. Mater. 2018, 28, 1802558-1802590.

(10) Penfold, T. J.; Dias, F. B.; Monkman, A. P. The Theory of Thermally Activated Delayed Fluorescence for Organic Light Emitting Diodes. Chem. Commun. 2018, 54, 3926-3935.

(11) Gibson, J.; Monkman, A. P.; Penfold, T. J. The Importance of Vibronic Coupling for Efficient Reverse Intersystem Crossing in Thermally Activated Delayed Fluorescence Molecules. ChemPhysChem 2016, 17, 2956-2961.

(12) Pander, P.; Dias, F. B. Photophysical Characterisation of Thermally Activated Delayed Fluorescence (TADF) Materials. Disp. Imaging 2017, 2, 249-263.

(13) Oh, C. S.; Pereira, D. d. S.; Han, S. H.; Park, H.-J.; Higginbotham, H. F.; Monkman, A. P.; Lee, J. Y. Dihedral Angle Control of Blue Thermally Activated Delayed Fluorescent Emitters through Donor Substitution Position for Efficient Reverse Intersystem Crossing. ACS Appl. Mater. Interfaces 2018, 10, 35420-35429.

(14) Dias, F. B.; Penfold, T. J.; Monkman, A. P. Photophysics of Thermally Activated Delayed Fluorescence Molecules. Methods Appl. Fluoresc. 2017, 5, 012001-012025.

(15) Kukhta, N. A.; Batsanov, A. S.; Bryce, M. R.; Monkman, A. P. Importance of Chromophore Rigidity on the Efficiency of Blue Thermally Activated Delayed Fluorescence Emitters. J. Phys. Chem. C 2018, 122, 28564-28575.

(16) Chen, T.; Zheng, L.; Yuan, J.; An, Z.; Chen, R.; Tao, Y.; Li, H.; Xie, X.; Huang, W. Understanding the Control of Singlet-Triplet Splitting for Organic Exciton Manipulating: A Combined Theoretical and Experimental Approach. Sci. Rep. 2015, 5, 10923-10933.

(17) Northey, T.; Stacey, J.; Penfold, T. J. The Role of Solid State Solvation on the Charge Transfer State of a Thermally Activated Delayed Fluorescence Emitter. J. Mater. Chem. C 2017, 5, 1100111009.

(18) Zhang, D.; Cai, M.; Bin, Z.; Zhang, Y.; Zhang, D.; Duan, L. Highly Efficient Blue Thermally Activated Delayed Fluorescent OLEDs 
with Record-low Driving Voltages Utilizing High Triplet Energy Hosts with Small Singlet-Triplet Splittings. Chem. Sci. 2016, 7, 3355-3363.

(19) Xie, G.; Chen, D.; Li, X.; Cai, X.; Li, Y.; Chen, D.; Liu, K.; Zhang, Q.; Cao, Y.; Su, S.-J. Polarity-Tunable Host Materials and Their Applications in Thermally Activated Delayed Fluorescence Organic Light-Emitting Diodes. ACS Appl. Mater. Interfaces 2016, 8, 2792027930 .

(20) dos Santos, P. L.; Ward, J. S.; Bryce, M. R.; Monkman, A. P. Using Guest-Host Interactions To Optimize the Efficiency of TADF OLEDs. J. Phys. Chem. Lett. 2016, 7, 3341-3346.

(21) Lee, I.; Lee, J. Y. Molecular Design of Deep Blue Fluorescent Emitters with 20\% External Quantum Efficiency and Narrow Emission Spectrum. Org. Electron. 2016, 29, 160-164.

(22) Ward, J. S.; Nobuyasu, R. S.; Fox, M. A.; Batsanov, A. S.; Santos, J.; Dias, F. B.; Bryce, M. R. Bond Rotations and Heteroatom Effects in Donor-Acceptor-Donor Molecules: Implications for Thermally Activated Delayed Fluorescence and Room Temperature Phosphorescence. J. Org. Chem. 2018, 83, 14431-14442.

(23) Chen, C.; Huang, R.; Batsanov, A. S.; Pander, P.; Hsu, Y.-T.; Chi, Z.; Dias, F. B.; Bryce, M. R. Intramolecular Charge Transfer Controls Switching Between Room Temperature Phosphorescence and Thermally Activated Delayed Fluorescence. Angew. Chem., Int. Ed. 2018, 57, 16407-16411.

(24) Etherington, M. K.; Franchello, F.; Gibson, J.; Northey, T.; Santos, J.; Ward, J. S.; Higginbotham, H. F.; Data, P.; Kurowska, A.; Dos Santos, P. L.; Graves, D. R.; Batsanov, A. S.; Dias, F. B.; Bryce, M. R.; Penfold, T. J.; Monkman, A. P. Regio- and Conformational Isomerization Critical to Design of Efficient Thermally-Activated Delayed Fluorescence Emitters. Nat. Commun. 2017, 8, 14987-14997.

(25) Dias, F. B.; Santos, J.; Graves, D. R.; Data, P.; Nobuyasu, R. S.; Fox, M. A.; Batsanov, A. S.; Palmeira, T.; Berberan-Santos, M. N.; Bryce, M. R.; Monkman, A. P. The Role of Local Triplet Excited States and D-A Relative Orientation in Thermally Activated Delayed Fluorescence: Photophysics and Devices. Adv. Sci. 2016, 3, 1600080-1600089.

(26) Pander, P.; Motyka, R.; Zassowski, P.; Etherington, M. K.; Varsano, D.; da Silva, T. J.; Caldas, M. J.; Data, P.; Monkman, A. P. Thermally Activated Delayed Fluorescence Mediated through the Upper Triplet State Manifold in Non-Charge-Transfer Star-Shaped Triphenylamine-Carbazole Molecules. J. Phys. Chem. C 2018, 122, 23934-23942.

(27) Numata, M.; Yasuda, T.; Adachi, C. High Efficiency Pure Blue Thermally Activated Delayed Fluorescence Molecules Having 10HPhenoxaborin and Acridan Units. Chem. Commun. 2015, 51, 94439446.

(28) Aydemir, M.; Xu, S.; Chen, C.; Bryce, M. R.; Chi, Z.; Monkman, A. P. Photophysics of an Asymmetric Donor-Acceptor-Donor' TADF Molecule and Reinterpretation of Aggregation-Induced TADF Emission in These Materials. J. Phys. Chem. C 2017, 121, 1776417772.

(29) Rothe, C.; Monkman, A. P. Triplet Exciton Migration in a Conjugated Polyfluorene. Phys. Rev. B: Condens. Matter Mater. Phys. 2003, 68, 075208-075218.

(30) Haase, N.; Danos, A.; Pflumm, C.; Morherr, A.; Stachelek, P.; Mekic, A.; Brütting, W.; Monkman, A. P. Kinetic Modeling of Transient Photoluminescence from Thermally Activated Delayed Fluorescence. J. Phys. Chem. C 2018, 122, 29173-29179.

(31) dos Santos, P. L.; Dias, F. B.; Monkman, A. P. Investigation of the Mechanisms Giving Rise to TADF in Exciplex States. J. Phys. Chem. C 2016, 120, 18259-18267.

(32) Nobuyasu, R. S.; Ren, Z.; Griffiths, G. C.; Batsanov, A. S.; Data, P.; Yan, S.; Monkman, A. P.; Bryce, M. R.; Dias, F. B. Rational Design of TADF Polymers Using a Donor-Acceptor Monomer with Enhanced TADF Efficiency Induced by the Energy Alignment of Charge Transfer and Local Triplet Excited States. Adv. Opt. Mater. 2016, 4, 597-607.

(33) dos Santos, P. L.; Ward, J. S.; Batsanov, A. S.; Bryce, M. R.; Monkman, A. P. Optical and Polarity Control of Donor-Acceptor Conformation and Their Charge-Transfer States in Thermally
Activated Delayed-Fluorescence Molecules. J. Phys. Chem. C 2017, $121,16462-16469$

(34) Dias, F. B.; Santos, J.; Graves, D. R.; Data, P.; Nobuyasu, R. S.; Fox, M. A.; Batsanov, A. S.; Palmeira, T.; Berberan-Santos, M. N.; Bryce, M. R.; Monkman, A. P. The Role of Local Triplet Excited States and D-A Relative Orientation in Thermally Activated Delayed Fluorescence: Photophysics and Devices. Adv. Sci. 2016, 3, 1600080-1600089.

(35) Zhang, J.; Ding, D.; Wei, Y.; Xu, H. Extremely Condensing Triplet States of DPEPO-Type Hosts through Constitutional Isomerization for High-Efficiency Deep-Blue Thermally Activated Delayed Fluorescence Diodes. Chem. Sci. 2016, 7, 2870-2882.

(36) Han, S. H.; Lee, J. Y. Spatial Separation of Sensitizer and Fluorescent Emitter for High Quantum Efficiency in Hyperfluorescent Organic Light-Emitting Diodes. J. Mater. Chem. C 2018, 6, 1504-1508.

(37) Kim, G. W.; Bae, H. W.; Lampande, R.; Ko, I. J.; Park, J. H.; Lee, C. Y.; Kwon, J. H. Highly Efficient Single-Stack Hybrid Cool White OLED Utilizing Blue Thermally Activated Delayed Fluorescent and Yellow Phosphorescent Emitters. Sci. Rep. 2018, 8, 16263-16273.

(38) Schmidt, T. D.; Lampe, T.; Sylvinson, M. R. D.; Djurovich, P. I.; Thompson, M. E.; Brütting, W. Emitter Orientation as a Key Parameter in Organic Light-Emitting Diodes. Phys. Rev. Appl. 2017, 8, 037001037028.

(39) Woo, S.-J.; Kim, Y.; Kwon, S.-K.; Kim, Y.-H.; Kim, J.-J. Phenazasiline/Spiroacridine Donor Combined with Methyl-Substituted Linkers for Efficient Deep Blue Thermally Activated Delayed Fluorescence Emitters. ACS Appl. Mater. Interfaces 2019, 11, 71997207. 